${ }^{5}$ Sumner, J. B., J. Biol. Chem., 69, 435 (1926); Ergebnisse der Enzymforschung, 1, 295-301, Leipzig (1932); Sumner, J. B., and Poland, L. O., Proc. Soc. Exptl. Biol. Med., 30, 553 (1933).

' Hopkins, F. G., J. Biol. Chem., 84, 269 (1929); Pirie, N. W., Biochem. J., 25, 614 (1931).

${ }^{7}$ Hellerman, L., and Newman, M. D., J. Am. Chem. Soc., 54, 2859 (1932).

\title{
THE ELECTRON-DIFFRACTION INVESTIGATION OF THE STRUCTURE OF MOLECULES OF METHYL AZIDE AND CARBON SUBOXIDE*
}

\section{By L. O. Brockway and Linus Pauling}

\section{Gates Chemical laboratory, California Institute of Technology}

Communicated August 8, 1933

We have applied the electron-diffraction method of studying the structure of gas molecules, developed by Mark and Wierl and briefly described by us in a recent paper, ${ }^{1}$ in the determination of the atomic configuration and interatomic distances in methyl azide and carbon suboxide molecules. The results of the investigation are given in the following paragraphs, together with a discussion of the information they provide regarding the electronic structure of these molecules.

Methyl Azide.-Two distinct structural formulas have been proposed for methyl azide, $\mathrm{CH}_{3} \mathrm{~N}_{3}$; in one the nitrogen atoms form a three-membered ring, and in the other an open chain. It is known that the azide ion has a linear configuration in crystals of sodium azide and potassium azide, ${ }^{2}$ but it has sometimes been contended that this configuration may not be retained in organic azides. Other methods of investigation have not led to a decision, the electric moment measurements, for example, being compatible with either structure. ${ }^{3}$ Our results show the azide group in methyl azide to be linear.

Each of five electron-diffraction photographs of methyl azide shows only one apparent maximum within the $6^{\circ}$ angular range allowed by the dimensions of the apparatus. This maximum is exceedingly sharp and distinct, and is susceptible to measurements of greater than the usual accuracy. The average measured value for $\frac{\sin \theta / 2}{\lambda}$ for it is 0.470 , and that for the welldefined apparent intensity minimum is 0.371 . These values are to be compared with intensity curves calculated for various models, as described in Reference 1.

Reasonable Lewis electronic structures in which the nitrogen atoms form a ring are the following: 


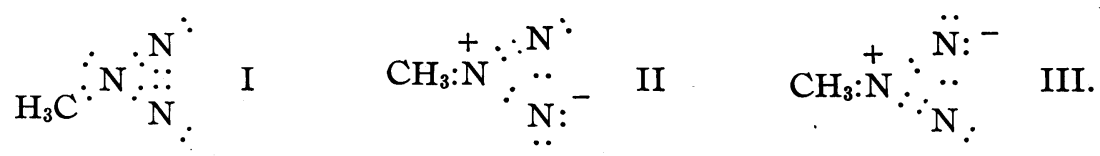

Since these structures contain the same total number of bonds and none of the atoms is doubly charged, we would expect that the molecule in its normal state would resonate among all three structures, their contributions being about equal. The inclusion of II and III in the group of resonating structures would require the methyl group to be coplanar with the ring. We shall call this structure A.

A.<smiles>CN1N=N1</smiles>

Coplanar III
Coplanar Structure I alone

Complete resonance among I, II and

B. $\mathrm{H}_{3} \mathrm{C}-\mathrm{N}_{\mathrm{N}}^{\mathrm{N}}$

C. Same as $\mathrm{B}$, but with $\mathrm{C}-\mathrm{N}$ to $\mathrm{N}-\mathrm{N}$ bond angles $133^{\circ} 30^{\prime}$

D. Same as C, but with bond angles $109^{\circ} 28^{\prime}$.

Calculations were also made for the less probable structures B, C and D (Fig. 1a). In each of these the interatomic distances are given by the type of bond and the atoms involved:4 $1.47 \AA$ for $\mathrm{C}-\mathrm{N}, 1.40 \AA$ for $\mathrm{N}-\mathrm{N}$, $1.26 \AA$ for $\mathrm{N}=\mathrm{N}$ and $1.06 \AA$ for $\mathrm{C}-\mathrm{H}$. The four carbon bonds are assumed to make angles of $109^{\circ} 28^{\prime}$ with each other. Interference between hydrogen and nitrogen atoms is neglected.

Reasonable electronic structures for a nitrogen chain are the following:

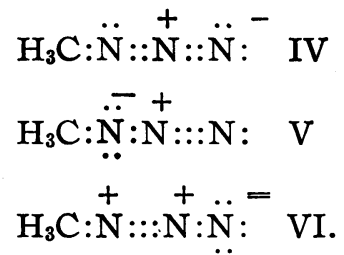

(In addition to these important structures, structures of the type $\mathrm{H}_{3} \mathrm{C}: \ddot{\mathrm{N}}: \mathrm{N}:: \ddot{\mathrm{N}}$ : ,etc., would contribute to a small amount, without changing the interatomic distances or bond angles.) We expect structures IV and V to contribute equally to the normal state of the molecule, since for them the total number of bonding electron pairs is the same and the arrangement of formal charges is nearly the same. Structure VI should be considerably less important because of the juxtaposition of atoms with charges of the same sign. Inasmuch as each of these structures corresponds to a col- 
linear arrangement of the nitrogen atoms in the azide group, the only bond angle varied in the structures for which calculations were made is that between the $\mathrm{C}-\mathrm{N}$ bond and the axis of the azide group. The structures considered are the following, the corresponding intensity curves being given in figure $1 b$ :
E. $\mathrm{H}_{3} \mathrm{C}-\mathrm{N} \equiv \mathrm{N} \equiv \mathrm{N} \quad$ Collinear
Complete resonance
F.

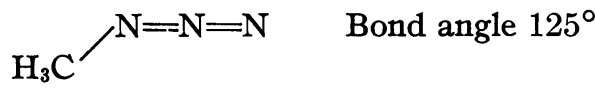 among IV, V and VI
G.

$\mathrm{H}_{3} \mathrm{C}^{\prime}=\mathrm{N}=\mathrm{N} \quad$ Bond angle $125^{\circ}$
Partial resonance be- tween IV and $\mathrm{V}$, with $\mathrm{N} \rightleftharpoons \mathrm{N}=1.16$ $\AA$
$\mathrm{H}-100^{\circ}{ }_{\mathrm{H}_{3} \mathrm{C}} / \mathrm{N}=\mathrm{N} \equiv \mathrm{N} \quad$ Bond angle as given
Complete resonance between IV and V
$\mathrm{H}-125^{\circ}$
$\mathrm{H}-129^{\circ} 10^{\prime}$
$\mathrm{H}-136^{\circ} 41^{\prime}$
$\mathrm{H}-150^{\circ}$
Structure IV

The interatomic distances used are those mentioned above, and $\mathrm{N} \equiv \mathrm{N}=$ $1.10 \AA$.

The qualitative comparison of the calculated curves of figures $1 a$ and $1 b$ and the observed photographs, showing a sharp maximum and minimum, leads immediately to the elimination of $\mathrm{E}$. The quantitative comparison may be conveniently made by combining the assumed $\mathrm{N}=\mathrm{N}$ interatomic distance of $1.26 \AA$ with the observed $\frac{\sin \theta / 2}{\lambda}$ values for the minimum and maximum to obtain "observed" values of $x=2 \cdot 1.26 \cdot \frac{\sin \theta / 2}{\lambda}$. This procedure is accepted as reliable because the atomic radii used have been verified by comparison with data for a large number of substances; and any analysis taking into account the effect of resonance on interatomic distance which gave values appreciably different from those obtained with the aid of the table would be rejected. The narrow shaded regions in the figures correspond to the observed position of the maximum with limits of error of $\pm 2 \%$. The shaded region for the first minimum extends from the "observed" value of $x$ to a value $10 \%$ smaller, inasmuch as we have found that for other substances investigated the visually estimated ring diameter for the first minimum is uniformly about 8 or $10 \%$ larger than 
the value given by calculation. Any structure for which the calculated maximum and minimum lie outside of the shaded regions is definitely eliminated. It is seen that all of the ring-type structures are ruled out; as

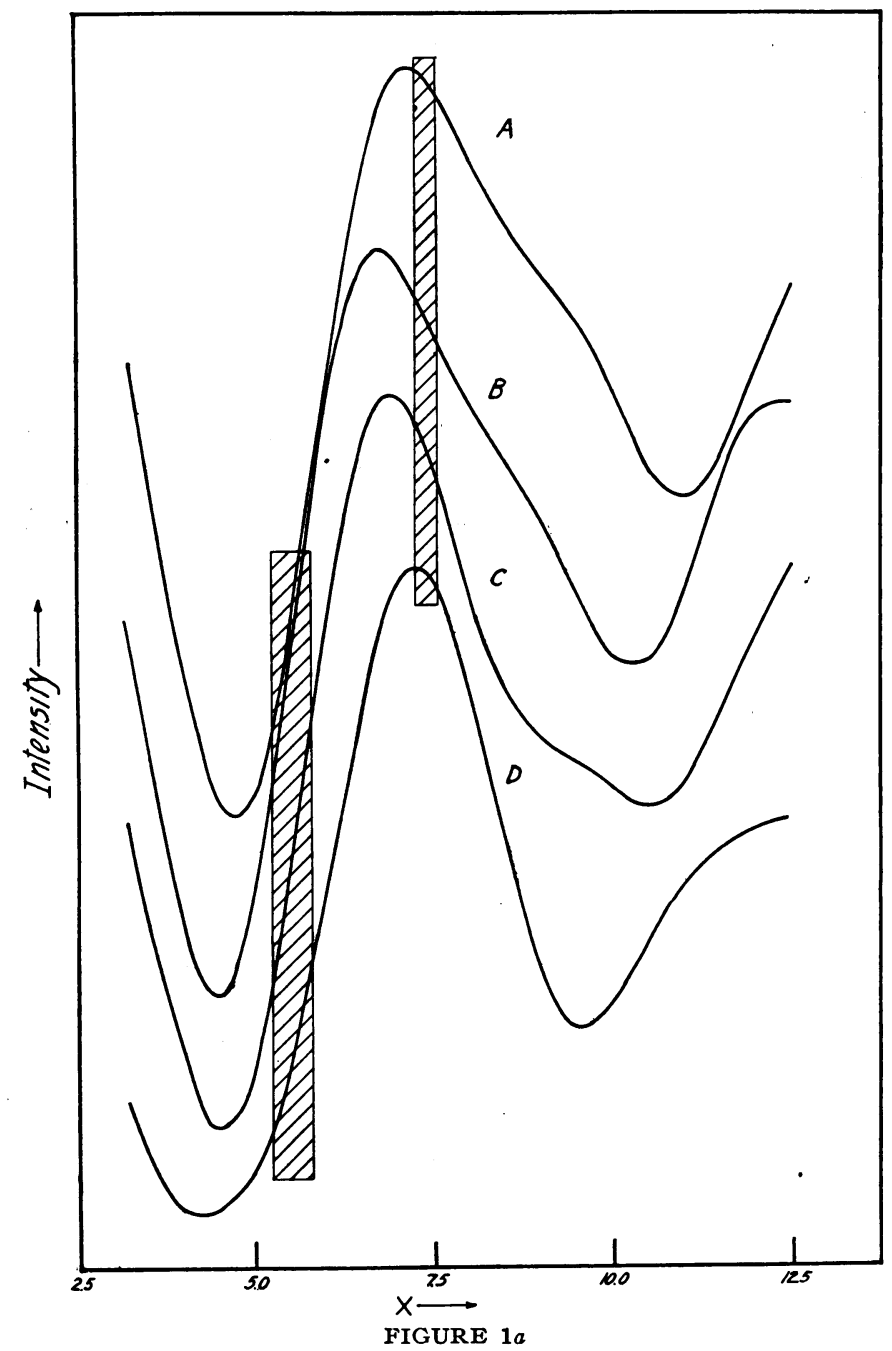

Theoretical intensity curves for cyclic structures of methyl azide.

well as $\mathrm{F}$ and $\mathrm{H}-100^{\circ}$. This leaves $\mathrm{G}$, representing partial resonance between IV and $\mathrm{V}$, and $\mathrm{H}-125^{\circ}$ to $\mathrm{H}-150^{\circ}$, representing complete resonance between these structures. While the experimental agreement with $\mathrm{G}$ is about as good as with $\mathrm{H}$, the probability of complete resonance between IV and V is very high, since the corresponding structures contribute 


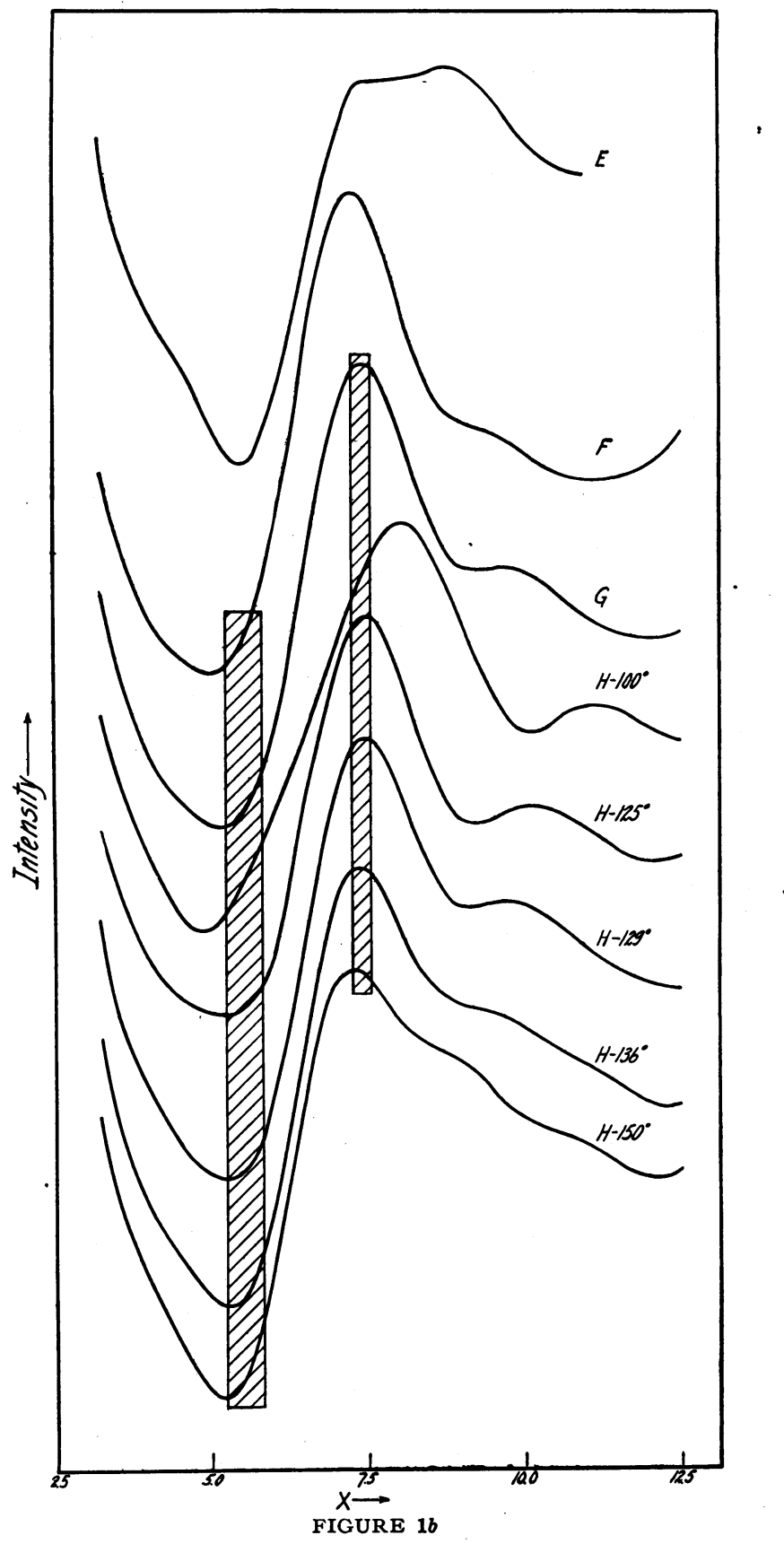

Theoretical intensity curves for chain structures of methyl azide. 
equally in the cases of $\mathrm{CO}_{2}$ and $\mathrm{N}_{2} \mathrm{O}$. The atomic arrangement of the normal state of methyl azide is thus found to be

$1.261 .10 \AA$

$\mathrm{H}_{3} \mathrm{C}$

$\mathrm{N}=\mathrm{N} \equiv \mathrm{N}$, with the

bond angle equal to $135^{\circ} \pm 15^{\circ}$. The uncertainty in the experimental verification of the interatomic distances is not larger than $\pm 2 \%$. The value found for the bond angle is close to that for a tetrahedral atom, $125^{\circ}$. The arrangement corresponds to resonance of the molecule among the two principal structures IV and V, with structure VI making no significant contribution. This supports the suggestion, originally advanced ${ }^{5}$ to account for the non-contribution of the structure $: \ddot{\mathrm{N}}: \stackrel{+}{\mathrm{N}}:: \stackrel{+}{\mathrm{O}}^{+}$to the normal state of nitrous oxide, that no important part in the normal state of molecules is played by structures containing adjacent atoms with electrical charges of the same sign.

Carbon Suboxide.-Both a ring and a chain structure have been proposed also for carbon suboxide:<smiles>O=C1C=CO1</smiles>

and<smiles>O=C=C=C=O</smiles>

The ring structure is extremely improbable because the bond angles for acetylene-type carbon atoms should be $180^{\circ}$, and the strains involved in the four-membered ring would make the molecule very unstable. We have hence not considered this structure. The most important electronic structures for the chain model are<smiles>O=C=CC(=O)C=CC(=O)C=CC(=O)C=CC(=O)C=CC(=O)C=O</smiles>

Of these the first three lead to a completely collinear arrangement of the atoms, so that we may safely assume all bond angles to be $180^{\circ}$. Resonance among all five structures leads to a model with distances corresponding to triple bonds throughout $(\mathrm{C} \equiv \mathrm{C}=1.22 \AA, \mathrm{C} \equiv \mathrm{O}=1.13 \AA)$. The same atomic configuration results from complete resonance among I, II 
and III only. Structure I alone leads to $\mathrm{C}=\mathrm{C}=1.38 \AA$ and $\mathrm{C}=\mathrm{O}=$ $1.28 \AA$, whereas partial resonance of I with II and III would lead to intermediate values, the ratio of the carbon-carbon to carbon-oxygen distance remaining constant (equal to 1.08). Curve $A$ in figure 2 consequently

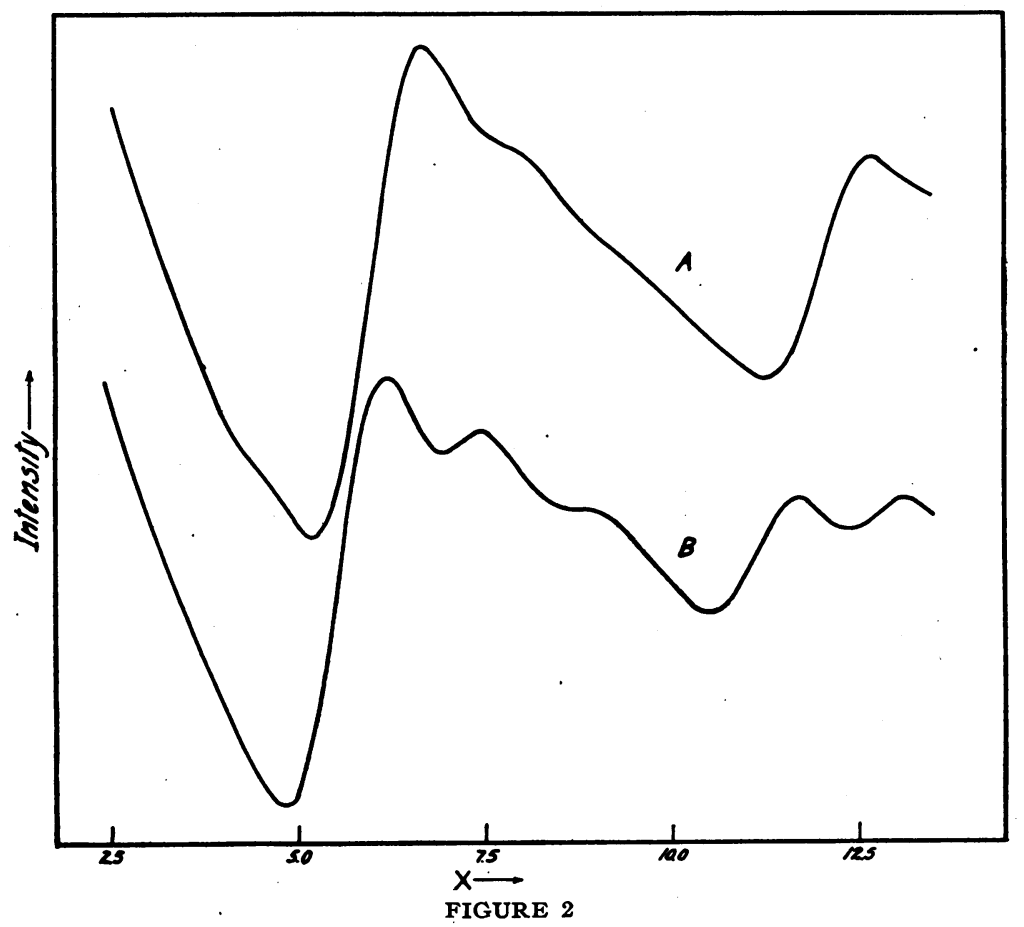

Theoretical intensity curves for carbon suboxide.

represents all of these models, the parameter $x$ being equal to the product of $\frac{\sin \theta / 2}{\lambda}$ and the carbon-oxygen distance. Curve $B$ represents resonance of I with IV and V.

The photographs show two distinct rings. There is no indication of the step-wise decrease in intensity following the first maximum required by $B$; therefore this model is eliminated. The quantitative comparison of the two minima and two maxima with curve $A$ is given in table 1 .

\begin{tabular}{llrc} 
& \multicolumn{2}{c}{ TABLE 1 } & \\
& $\frac{\sin \theta / 2}{\lambda}$ & $x$ & $a$ \\
1st min. & 0.367 & 5.18 & 1.12 \\
1st max. & 0.438 & 6.66 & 1.21 \\
2nd min. & 0.756 & 11.22 & 1.18 \\
2nd max. & 0.849 & 12.70 & 1.19
\end{tabular}


The agreement in the values of the carbon-oxygen distance $a$ is satisfactory, taking into account the usual 8 or $10 \%$ deviation shown by the first minimum, and provides strong support for the model. The averaged value of $a$ is $1.193 \AA$ (neglecting the first minimum), which becomes $1.20 \AA$ when the small density correction required by comparison with the carbon tetrachloride standard photographs is made. The interatomic distances hence have the values

$$
\mathrm{C} \text { to } \mathrm{O}=1.20 \pm 0.02 \AA, \quad \mathrm{C} \text { to } \mathrm{C}=1.30 \pm 0.02 \AA \text {, }
$$

which are just midway between the double-bond and triple-bond values. Consequently the most important contribution to the normal state of the molecule is made by structure I; there is, however, incomplete resonance to structures II and III, to an amount sufficient to decrease the interatomic distances appreciably. The elimination of model $B$ indicates that structures IV and V contribute still less than II and III. The bonds in the molecule have properties intermediate between double and triple bonds, and the normal state might be conveniently represented by the formula $\mathrm{O} \equiv \mathrm{C} \equiv \mathrm{C} \equiv \mathrm{C} \equiv \mathrm{O}$.

We are indebted to Dr. G. W. Wheland for preparing the methyl azide and carbon suboxide used in the experiments.

* Contribution from Gates Chemical Laboratory, California Institute of Technology, No. 353.

${ }^{1}$ Brockway, L. O., and Pauling, L., these Proceedings, 19, 68 (1933).

${ }^{2}$ Hendricks, S. B., and Pauling, L., Jour. Amer. Chem. Soc., 47, 2904 (1925).

${ }^{3}$ Sidgwick, N. V., Sutton, L. E., and Thomas, W., J. Chem. Soc., 406 (1933).

4 Pauling, L., these Proceedrngs, 18, 293 (1932).

5 Pauling, L., Ibid., 18, 498 (1932). 